\title{
Sacral Nerve Modulation Has No Effect on the Postprandial Response in Irritable Bowel Syndrome
}

This article was published in the following Dove Press journal:

Clinical and Experimental Gastroenterology

\author{
Janne Fassov (D) \\ Donghua Liao ${ }^{2}$ \\ Christina Brock (iD) ${ }^{3}$ \\ Lilli Lundby 4 \\ Søren Laurberg ${ }^{4}$ \\ Klaus Krogh' \\ 'Department Of Hepatology and \\ Gastroenterology, Aarhus University \\ Hospital, Aarhus, Denmark; ${ }^{2}$ GIOME \\ Academia, Department of Clinical \\ Medicine, Aarhus University, Aarhus, \\ Denmark; ${ }^{3}$ Mech-Sense, Department of \\ Gastroenterology and Hepatology, \\ Aalborg University Hospital and Clinical \\ Institute, Faculty of Health Sciences \\ Aalborg University, Aalborg, Denmark; \\ ${ }^{4}$ Department of Surgery, Aarhus \\ University Hospital, Aarhus, Denmark
}

Correspondence: Janne Fassov Department of Hepatology and Gastroenterology, Aarhus University Hospital, Palle Juul-Jensens Boulevard 99, Aarhus N 8200, Denmark

Tel +4526756602

Email janfas@rm.dk

\begin{abstract}
Purpose: Irritable bowel syndrome is a common gastrointestinal disorder with a global prevalence of approximately $11 \%$. Onset or worsening of symptoms following digestion is one of the characteristics of the condition. The present study aimed at evaluating the postprandial sensory and motor response before and after treatment with sacral nerve modulation.

Patients and Methods: Twenty-one irritable bowel syndrome patients, 12 diarrheapredominant and 9 mixed, were eligible for a 6-week sacral nerve modulation test period. Patients were investigated with multimodal impedance planimetry including a standardized meal at baseline and at the end of 2 weeks of suprasensory stimulation embedded in the 6-week sacral nerve modulation period.
\end{abstract}

Results: There was no statistical significant difference in the sensory response to heat or cold before and after sacral nerve modulation, $p>0.05$. At baseline, wall tension increased after the meal (mean 124.79 [range 82.5 to 237.3] mmHg.mm before the meal, mean 207.76 [range, 143.5 to 429 ] $\mathrm{mmHg} . \mathrm{mm}$ after the meal), $\mathrm{p}=0.048$ indicating a postprandial response. During sacral nerve modulation, the postprandial increase in wall tension did not reach statistical significance (mean 86.79 [range 28.8 to 204.5] mmHg.mm before the meal, mean 159.71 [range 71.3 to 270.8 ] $\mathrm{mmHg} . \mathrm{mm}$ after the meal), $\mathrm{p}=0.277$. However, there was no statistically significant difference between the postprandial wall tension at baseline and during sacral nerve modulation, $\mathrm{p}=0.489$. Likewise, we found no difference between pressure or stretch ratio at baseline and during sacral nerve modulation, $\mathrm{p}>0.05$.

Conclusion: Sacral nerve modulation does not exert its positive treatments effects in diarrhea-predominant and mixed irritable bowel syndrome through a modulation of the postprandial response.

Keywords: sacral nerve stimulation, sacral nerve modulation, postprandial response, gastrocolic response, irritable bowel syndrome

\section{Introduction}

Irritable bowel syndrome (IBS) is one of the most common gastrointestinal disorders and accounts for around one-third of gastrointestinal complaints in general practice. ${ }^{1}$ The syndrome consists of intermittent abdominal pain associated with defecation and a change in stool frequency and consistency currently defined by the Rome IV criteria. ${ }^{2}$ The etiology of IBS is unknown and therefore potential treatment is based on symptom reduction. Most IBS patients experience a postprandial onset or worsening of symptoms. ${ }^{3,4}$ Additionally, studies have found both an abnormal motor and sensory postprandial response in IBS patients. ${ }^{5-7}$

Much effort has been put in finding a treatment with a long-lasting effect managing as many of the IBS-specific symptoms as possible. Recent studies on sacral nerve 
modulation (SNM) for diarrhea-predominant and mixed IBS (IBS-D and IBS-M) have found a positive, sustained treatment effect regarding both IBS-specific symptoms and quality of life. ${ }^{8-10}$

$\mathrm{SMN}$ is administered through a surgically placed electrode in the sacral foramina of S3 or S4, that delivers a continuous low-intensity current to the sacral nerves. The electrode is connected to a neurostimulator on the ipsilateral higher buttock and the combined system is operated through a personal remote control. SNM is approved for fecal incontinence in Europe and the United States. Evidence suggests that the mode of action of SNM is by a direct efferent effect on the anal sphincter complex as well as modulation of afferent input to the sacral spinal cord and/or supraspinal centers. ${ }^{11-13}$

Multimodal impedance planimetry is a validated method for investigations of rectal sensitivity and biomechanical properties and has previously been described in detail. ${ }^{14}$ Moreover, the system has previously identified a hyperactive postprandial response in patients with low anterior resection syndrome following treatment for rectal cancer. ${ }^{15}$
The primary aim of the present study was to evaluate if SNM has an effect on the postprandial response in IBS-D and IBS-M patients.

\section{Patients and Methods}

\section{Patients}

The present study includes the IBS patients, whom between October 1, 2013 and January 31, 2018 were included in a randomized, double-blinded, cross-over study at our tertiary clinic to evaluate the treatment effect of SNM in IBS patients, (Figure 1). ${ }^{9}$ The diagnosis of IBS was performed according to the Rome III criteria, 12 were subtyped with diarrheapredominant IBS and 9 with mixed IBS. There was no evidence of lactose intolerance, celiac disease, or thyroid disease in any of the patients and all had undergone a normal endoscopy including biopsies. Patients were acquired to present with an average IBS-specific symptom score (Gastrointestinal Symptom Rating Scale - Irritable Bowel Syndrome (GSRS-IBS) version) of at least 40 points during a 3-week period prior to enrollment. Exclusion criteria

IBS-D and IBS-M patients referred for possible inclusion in the double-blinded, placebo-controlled crossover study $(n=67)$

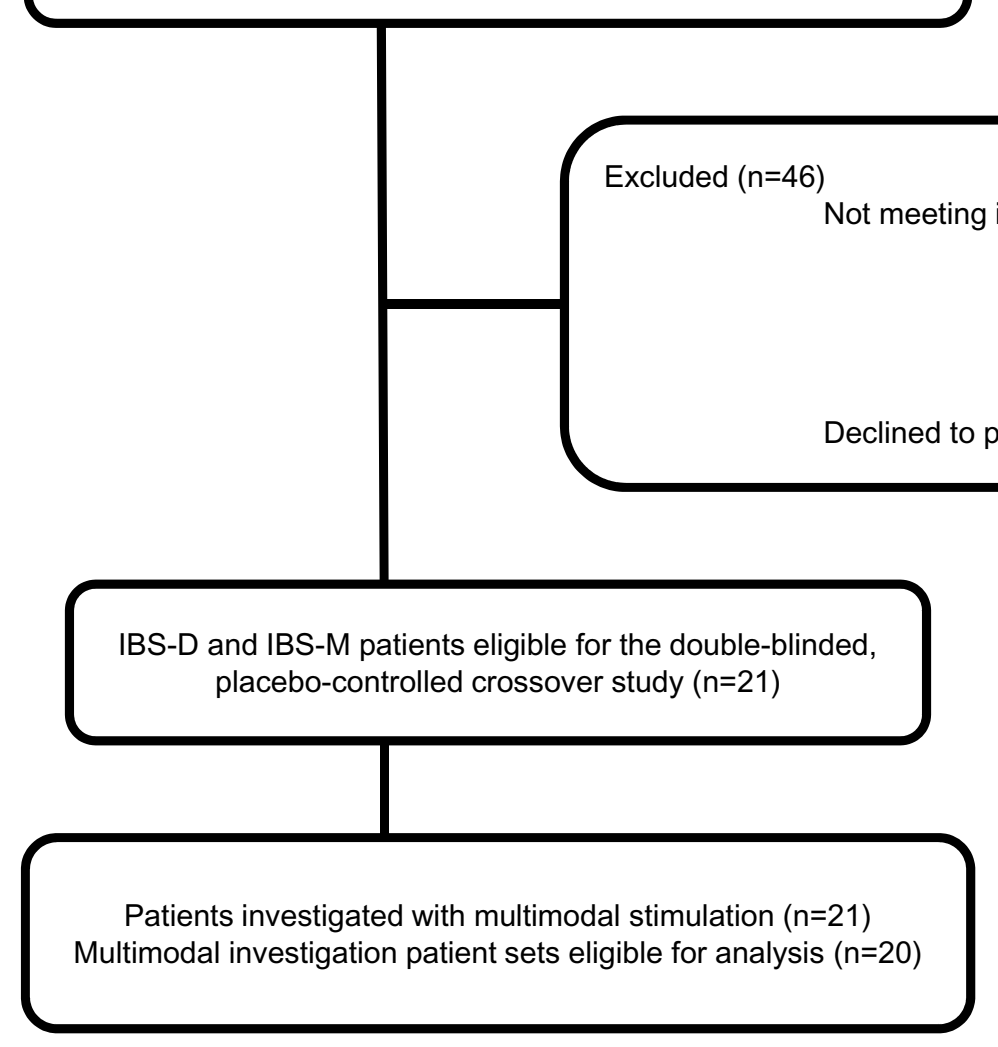

Figure I Patient cohort. 
included constipation-predominant IBS, severe psychological comorbidity, pregnancy, and age below 18 or above 70 .

\section{Study Design}

The design of the study was a 6-week SNM period consisting of a 4-week randomized, double-blinded, placebo-controlled crossover study testing subsensory stimulation versus no stimulation followed by a 2-week period of suprasensory stimulation. Patients were investigated with multimodal impedance planimetry at baseline before entering the study and at the very end of the 2-week suprasensory SNM period.

The study was approved by the Danish Ethics Committee (ID1-10-72-170-13) with every patient giving informed and written consent before inclusion as well as conducted in accordance with the Declaration of Helsinki. The study was registered at Clinicaltrials.gov, NCT01950715.

\section{Procedures}

\section{Sensory Assessment}

Before initiating multimodal impedance planimetry, patients were familiarized with the continuous electronic modified visual analog scale (VAS) ranging from 0 to 10 . The numbers equaled $0=$ no perception, $1=$ vague perception, $2=$ definite perception of mild sensation, $3=$ vague perception of moderate sensation, $4=$ definite perception of moderate sensation, $5=$ pain detection threshold (PDT), $6=$ slightly pain, $7=$ moderate pain detection threshold (MPDT), $8=$ medium pain intensity, $9=$ intense pain, and $10=$ unbearable pain. Earlier studies have proven the scale applicable evaluating visceral pain. ${ }^{16,17}$ The intensity of the sensory response was recorded continuously for each type of stimulus during which patients were informed to notify level 1, 3, 5, and 7. No stimulation went beyond VAS 7.

\section{Multimodal Stimulation}

Procedures were undertaken with a custom-designed multimodal probe. The non-compliant $30 \mu \mathrm{m}$ thick and $70 \mathrm{~mm}$ long polyurethane bag attached $2 \mathrm{~cm}$ under the tip had a maximum diameter of $11.5 \mathrm{~cm}$ and maximum recordable cross-sectional area (CSA) of $10.380 \mathrm{~mm}^{2}$.

An enema (Klyx; Ferring, Copenhagen, Denmark) was given thirty minutes prior to procedures. The patient thereafter lay in a bed following which, the multimodal probe was advanced through an anoscope (WelchAllyn, Skaneateles Falls, NY, USA) to a final position $18 \mathrm{~cm}$ from the anal verge. Stimulations then followed protocol in the given order; thermal, mechanical, standardized breakfast, mechanical.
For thermal stimulation, the bag was filled with $60 \mathrm{~mL}$ of $0.9 \%$ saline to ensure mucosal contact. Hereafter, $4{ }^{\circ} \mathrm{C}$ (cold) or $68{ }^{\circ} \mathrm{C}$ (heat) $0.9 \%$ saline were administered recirculating the saline from a closed circuit including either an ice-cubed filled container or a stirred water bath (Grant GD100; Grant Instruments, Cambridge, UK). The inlet rate of saline was set to $150 \mathrm{~mL} / \mathrm{min}$ controlled by a peristaltic pump (Type 110ACR; Ole Dich Instrumentmakers, Hvidovre, Denmark), which gave a temperature incline rate of $0.5{ }^{\circ} \mathrm{C} / \mathrm{s}$. Temperatures in the bag were at all times noted using a sensor (Buhl and Bønsøe AS, Virum, Denmark) and analyzed according to sensory levels (VAS 1-7).

For mechanical stimulation, the bag was filled with $0.9 \%$ saline $\left(37^{\circ} \mathrm{C}\right)$ at a continuous flow rate of $200 \mathrm{~mL} / \mathrm{min}$ administered by the peristaltic pump. Embedded in the probe, a sixelectrode impedance measuring system made real time recording of two CSA's. The system included two pairs of detection electrodes placed with an inter-distance of $2 \mathrm{~mm}$ measuring the CSA1 and CSA2. On each side of the pairs of detection electrodes an outer stainless steel excitation electrode was receiving a constant $\mathrm{AC}$ current from a connected impedance planimetry box (Ditens, A/S, Egaa, Denmark). The pressure was obtained by a low-compliance perfusion system (Edwards Lifesciences, Irvine, CA, USA) applying an external pressure transducer. For mechanical and sensory preconditioning, three distensions were performed and stopped at PDT (VAS 5). Hereafter, a single distension was performed and stopped at MPDT (VAS 7). CSA's were calculated as the mean of CSA1 and CSA2 $\left(\mathrm{mm}^{2}\right)$. Strain was defined as the wall circumferential length between minimum [the circumferential length at VAS 1] and maximum distension [circumferential length at VAS 7], divided by the length at minimum distension (unitless). CSA's, strain, and wall tension (mmHg.mm) were all analysed according to sensory levels (VAS 1-7). The preconditioning and the following single distension reaching MPDT was repeated after a standardized meal.

The standardized continental breakfast contained $1991 \mathrm{~kJ}$ ( $14.2 \%$ protein, $44.9 \%$ fat, and $40.9 \%$ carbohydrates). The meal was ingested after a minimum fast of 8 hours. The mechanical stimulation was repeated twenty minutes after consumption.

\section{Statistical Analyses}

The sample size calculation in the study was based on evaluating the clinical effect of SNM in the previously published, randomized, double-blinded, placebo-controlled crossover study. $^{9}$ 
For comparison of sensory data, we performed twoway repeated measures ANOVA. For evaluation of biomechanical properties, statistical testing was performed according to the distribution of data with a paired $t$-test or the Wilcoxon signed rank test.

Data are provided as median (25th percentile, 75th percentile) and mean \pm SD unless otherwise stated. For the statistical analysis, we used the software package SigmaPlot for Windows Version 11.0 (Systat Software, Inc, San Jose, CA, USA). P-values less than 0.05 were considered statistically significant.

Post hoc we performed power analysis for tension, pressure, and stretch based on results in the present study with $\alpha=0.05$.

\section{Results}

Of the 21 patients included in the randomized, doubleblinded, placebo-controlled crossover study, 20 were eligible for analysis completing both multimodal stimulation at baseline and at the end of the two weeks of suprasensory SNM, Figure 1. All patients had failed standard treatment including, dietary adjustments and lifestyle changes, conventional anti-diarrheal, in one case eluxadoline (released in Denmark in April 2017), conventional osmotic laxatives, and linaclotide. Data on the clinical effect of SNM has been reported previously. In summary, SNM significantly reduced pain and number of daily bowel movements, while the overall IBS-specific symptom score was only reduced with borderline significance $(p=0.0572)$. Fifteen out of 20 patients had a successful suprasensory stimulation period. ${ }^{9}$

For patient characteristics, see Table 1.

We observed no procedure-related adverse effects. VAS 7 was reached for all patients at any stimuli administered.

\section{Thermal Stimulation}

There was no difference in the sensory response to neither heat nor cold comparing multimodal stimulation at baseline and at the end of the suprasensory SNM period ( $>00.05$ ), Figure 2 .

\section{Mechanical Stimulation Verification of a Postprandial Response}

At baseline, median wall tension at VAS 1 increased significantly following the meal, 207.76 (range, 143.5 to 429) mmHg.mm, compared to before the meal, 124.79 (range 82.5 to 237.3 ) $\mathrm{mmHg} . \mathrm{mm}, \mathrm{p}=0.048$.
Table I Patient Characteristics

\begin{tabular}{|l|l|}
\hline & Study Group N=20 \\
\hline Age (years, median range) & $25(21,53)$ \\
Men/Women & $6 / 14$ \\
Diarrhoea-predominant/mixed IBS & $12 / 8$ \\
Baseline IBS-specific scores (median [range]) & \\
Total GSRS-IBS score & $58.5(41,77)$ \\
\hline Pain & $10(5,17)$ \\
Bloating & $16.5(7,21)$ \\
Constipation & $8(2,12)$ \\
Diarrhoea & $20.5(10,26)$ \\
Satiety & $6(2,14)$ \\
Total IBS-IS score & $132.5(48,163)$ \\
\hline Fatigue & $31.5(6,42)$ \\
Daily impaired activities & $37(17,49)$ \\
Sleep & $16(5,27)$ \\
Emotional distress & $29.5(7,35)$ \\
Eating habits & $21(9,28)$ \\
\hline
\end{tabular}

Note: Values are expressed as median and range.

Abbreviations: GSRS-IBS, Gastrointestinal Symptom Rating Scale - Irritable Bowel version; IBS-IS, Irritable Bowel Syndrome - Impact Scale.

\section{SNM and the Postprandial Response}

\section{Tension}

At the end of the suprasensory SNM period, we found no statistically significant difference when comparing median wall tension at VAS 1 before, 86.79 (range 28.8 to 204.5) mmHg.mm and after the meal, 159.71 (range 71.3 to 270.8) mmHg.mm, $p=0.277$. Nevertheless, there was no statistical significant difference when comparing the postprandial wall tension at VAS 1 at baseline and the end of the suprasensory SNM period, $\mathrm{p}=0.489$. This was also true for all other VAS levels ( $\mathrm{p}>0.05)$, Figure 3 .

\section{Pressure}

There was no statistically significant difference in the pressure changes from VAS 1 to VAS 7 when comparing postprandial data at baseline and at the end of the suprasensory SNM period $(\mathrm{p}=0.358)$.

\section{Stretch Ratio}

The stretch ratio at VAS 7 after the meal at baseline was not statistically significant different with the stretch ratio at VAS 7 after the meal at the end of the suprasensory SNM period $(\mathrm{p}=0.750)$, Figure 4 .

\section{Comparison of Difference Between Postprandial Changes at Baseline and During Suprasensory SNM}

There was no difference in the median tension difference at VAS $7(\mathrm{p}=0.818)$, the median pressure difference at 


\section{COLD}

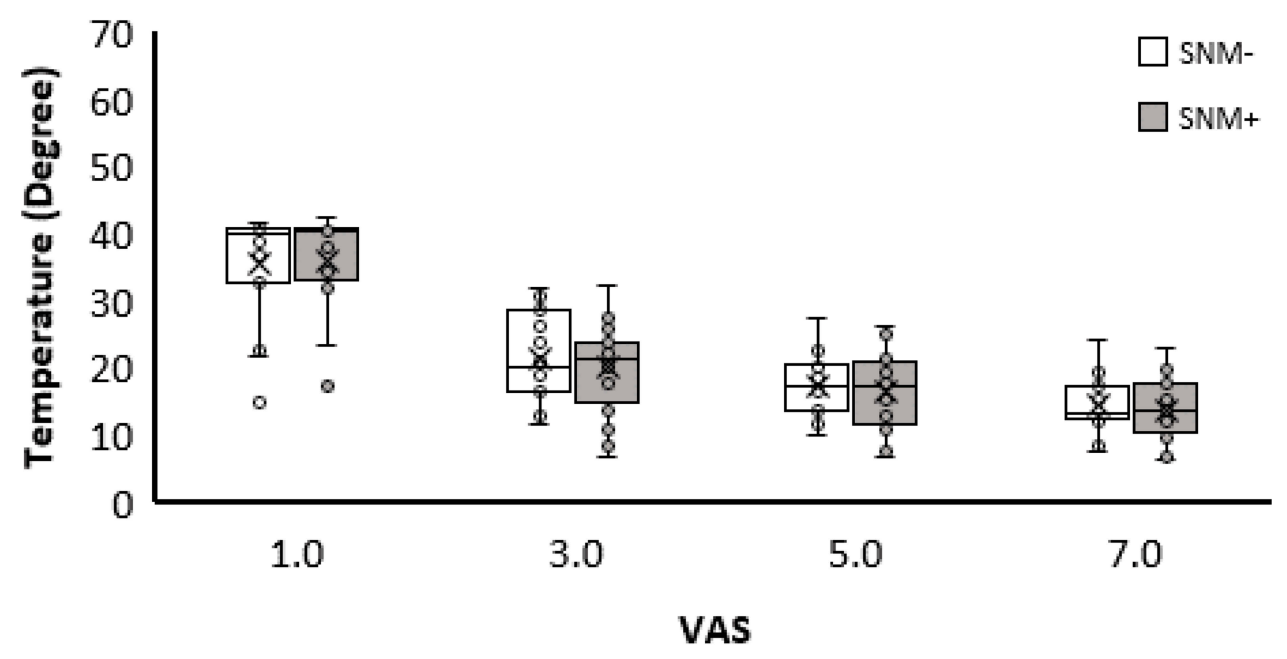

HEAT

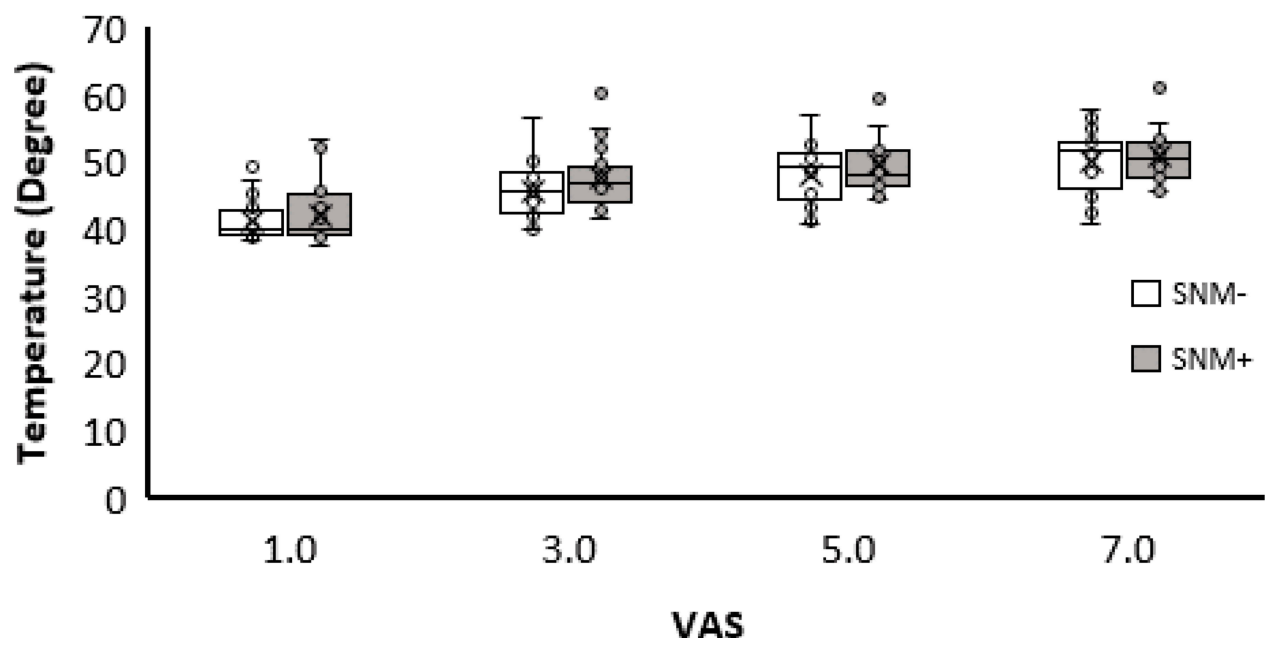

Figure 2 Change in temperature to Visual Analog Scale (VAS) score increase. $X$ marks the mean and the line in the box the median.

VAS $7(\mathrm{p}=0.541)$, or the stretch ratio difference at VAS 7 $(\mathrm{p}=0.971)$.

\section{Comparison of SNM Responders and Non-Responders}

Within the groups of SNM responders and non-responders, we found no difference in tension, pressure, or stretch when comparing baseline and suprasensory SNM data before and after the meal $(\mathrm{p}>0.05)$.

Neither did we find any significant difference in tension or pressure, when comparing SNM responders to non-responders at baseline and at the end of the SNM suprasensory stimulation period before and after the meal $(\mathrm{p}>0.05)$. We did observe a significant difference in mean stretch between SNM responders and nonresponders before the meal at both baseline $2.64 \pm 1.82$ vs $1.44 \pm 0.21, \mathrm{p}=0.04$ and in the end of the suprasensory stimulation period $3.00 \pm 2.07$ vs. $1.47 \pm 0.29, \quad \mathrm{p}=0.02$. Analysis of differences between groups were, however, non-significant $(\mathrm{p}>0.05)$.

\section{Power Analysis}

Given $\mathrm{n}=20$ and the observed differences and standard deviations, the calculated power was 0.81 for tension, 0.86 for pressure, and 0.84 for stretch. 


\section{Before meal}

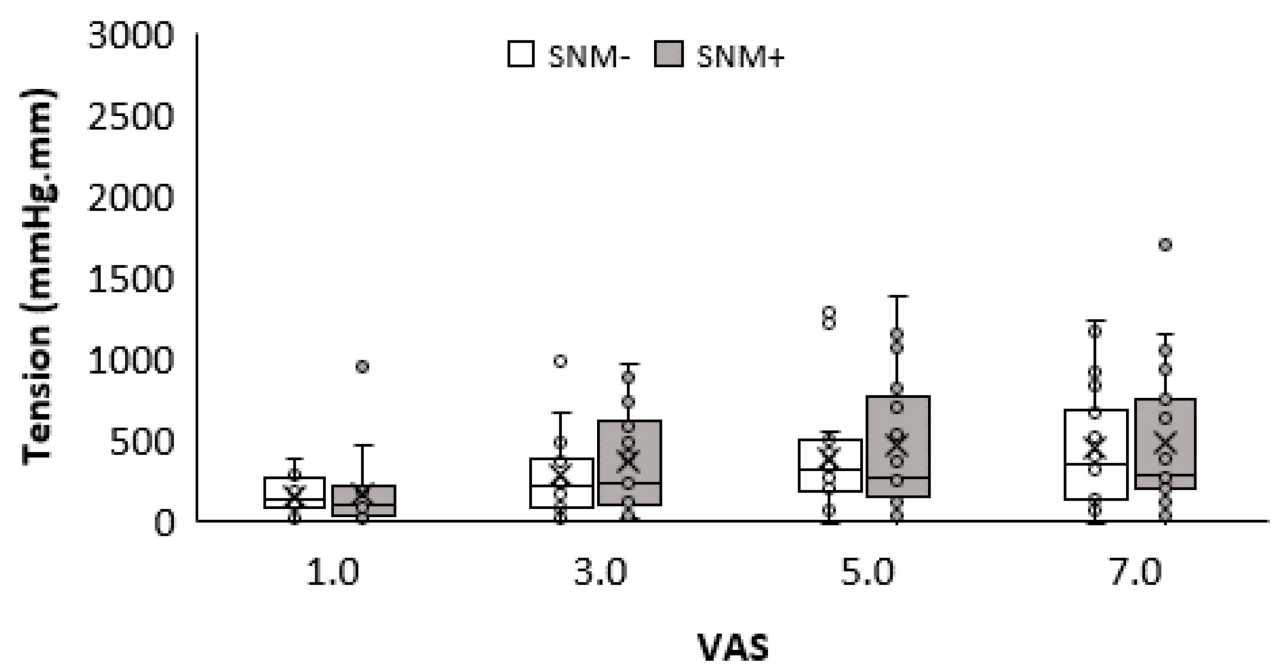

\section{After meal}

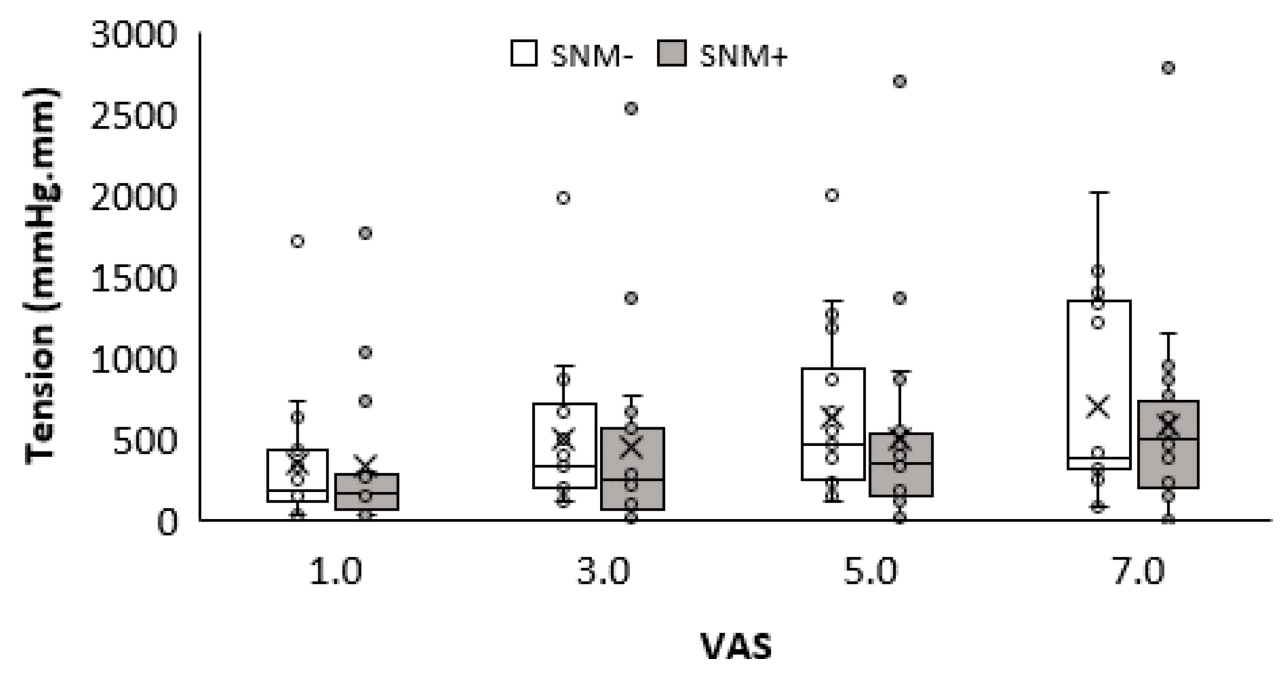

Figure 3 Change in stretch ratio to Visual Analog Scale (VAS) score increase. $X$ marks the mean and the line in the box the median.

\section{Discussion}

In this interventional study investigating the mechanism of action of SNM for IBS-D and IBS-M, we found no indications that SNM acts through modulation of the postprandial response. We did find the tension to increase at baseline after patients had consumed their meal indicating a postprandial response. However, although the tension levels recorded after the meal during the SNM period were lower, the differences did not reach statistical significance. Neither did we find any statistically significant differences when comparing pressure, tension, or stretch ratio at VAS 7 at baseline and at the end of the suprasensory SNM period. Additionally, there was no statistically significant difference when comparing the differences between changes in tension, pressure, or stretch ratio before and after the meal at baseline and at the end of the suprasensory SNM period. Subdividing the patients into SNM responders and non-responders we found no statistically significant differences regarding tension, pressure, or stretch within the groups. Comparing the groups, we surprisingly observed that SNM responders were significantly more compliant to stretch than non-responders before the meal both at baseline and at the end of the suprasensory stimulation period. The postprandial change was, however, nonsignificant. Whether this may be a positive, prognostic factor for a PNE success remains to be studied. 


\section{Before meal}

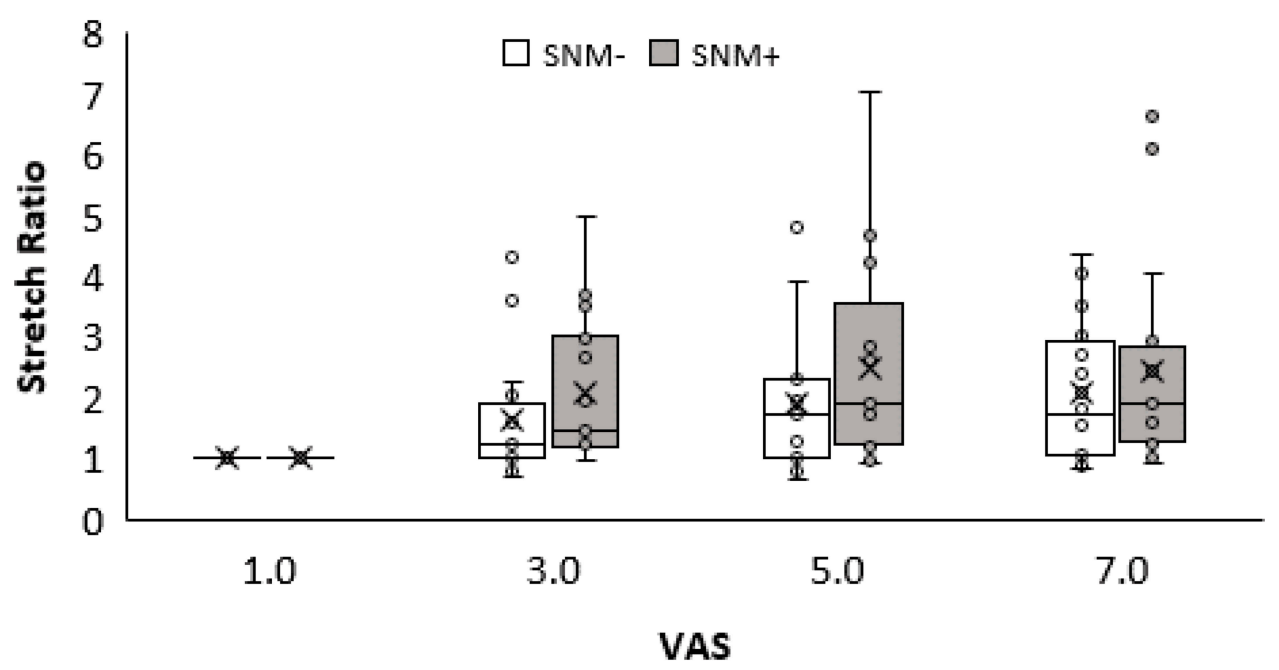

\section{After meal}

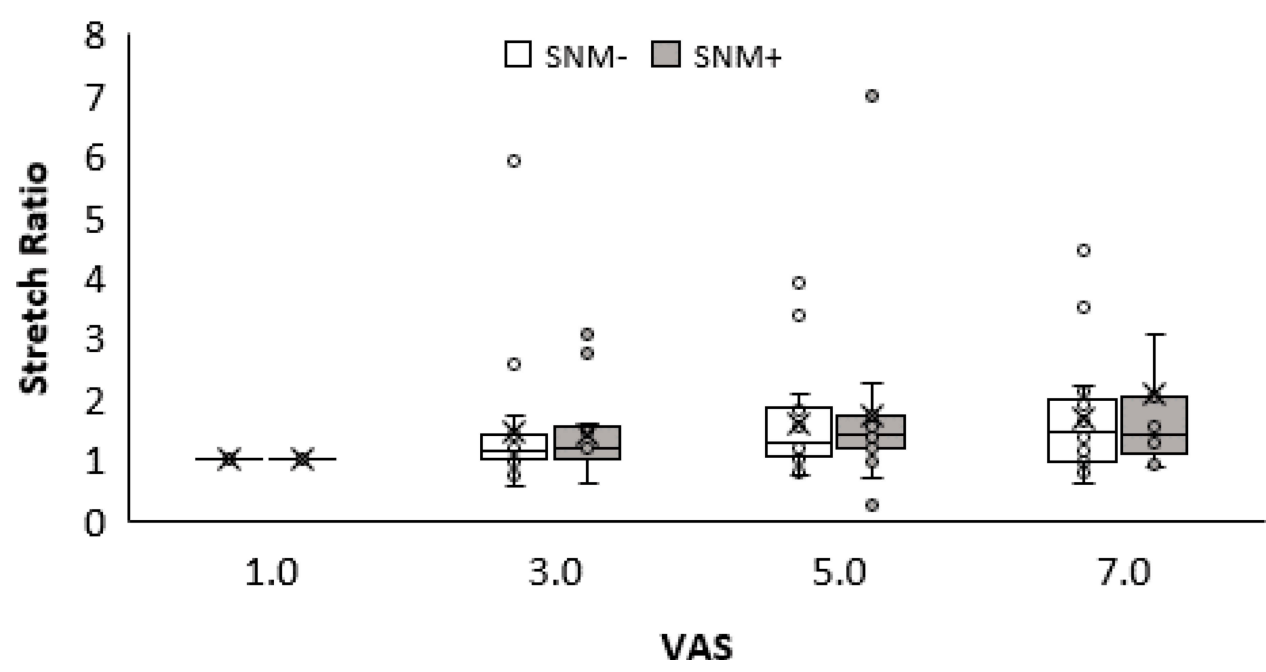

Figure 4 Change in tension to Visual Analog Scale (VAS) score increase. $X$ marks the mean and the line in the box the median.

Only few studies have investigated a given treatment's effect on the postprandial response in IBS patients. In accordance with our present data, those previous studies did not find that treatments, which reduced symptoms of IBS, did so by changing the postprandial response. Thus, Simrén and colleges found that hypnotherapy for IBS did not lower the threshold to perception of gas and discomfort after a duodenal lipid infusion. ${ }^{18}$ In addition, they observed significantly lower fasting balloon volumes (increased sigmoid tone) in the hypnotherapy group compared to the control group. In our study, patients were not instructed to report perceptions of gas, but only vague perception of moderate sensation, pain, and moderate pain. Common to both studies, the given treatment had no effect on the pain perceived by the patients following a meal. In another recent study by Simrén et al, authors found that the selective $5-\mathrm{HT}_{3}$ receptor antagonist, Alosetron, reduced pain following administration of duodenal lipids without having an effect on the postprandial response. $^{19}$

There are limitations to the present study. The sample size calculation was based on the number needed to evaluate the treatment effect of SNM for IBS-D and IBS-M in the previous published double-blinded, placebo-controlled crossover study. ${ }^{9}$ However, subsequent power analysis for tension, pressure, and stretch found a power of $0.81,0.86$, 
and 0.84 respectively, indicating the sample size was sufficient to detect the specified effect.

Furthermore, the IBS patients in the study were challenged by consuming the entire standardized meal within their given time period. Care was taken that the individual consumed meal portions were comparable at baseline and at the end of the suprasensory SNM period and we did see a postprandial response at baseline. Finally, the study relies on accurate patient reporting based on the visual analog scale, although, multimodal stimulation data has previously proven to be responsive to change. ${ }^{14}$

The mechanism of action of SNM may be a combination of a direct efferent effect on the anal sphincter complex and a modulation of afferent input to the sacral spinal cord and/or supraspinal centers. ${ }^{11-13}$ Looking into the mode of action of SNM for IBS, our group has previously published papers investigating the effect on gastrointestinal motility, rectal sensitivity and biomechanical properties. We found no effect of SNM on gastric emptying or small intestinal transit patterns in neither the fasting nor the postprandial state. ${ }^{20} \mathrm{We}$ did, however, observe a relaxation of the rectal wall associated with a reduced IBS-specific symptom score and a sensitization to stretch associated with a reduced constipation domain score in the IBS-specific symptom questionnaire. $^{21}$ We were not able to reproduce those data in the present study. This may be due to a lower number of patients having a positive treatment effect of SNM in the present study (fifteen versus twenty). It could also be due to the SNM treatment length before investigation. Where the twenty-one patients in the initial study had received SNM for a median of 5.8 months (1-20 months) before multimodal impedance planimetry, it was only for 2 weeks in the present study. However, we did show a positive effect on symptoms in both studies.

Tillisch et al have previously observed that patients with IBS have a greater than normal engagement of cerebral regions associated with emotional arousal and endogenous pain modulation during rectal distensions. ${ }^{22}$ Furthermore, Haas et al have found impaired cerebral processing of rectal stimuli and an altered cortical processing of anal distention's in fecal incontinence. ${ }^{23}$ Finally, Giani and colleagues have reported that prolonged latency of cerebral somatosensory evoked potentials is a predictor of a positive treatment effect of SNM for fecal incontinence. Therefore, they concluded, that SNM might act on a cortical level via modulation of the afferent pathway from the spinal nerves to the sensory cortex. ${ }^{24}$ Thus, the authors propose that future studies should investigate whether the positive treatment effect of SNM for IBS is driven by changes in cerebral function.

Further, it should be taken into consideration that Griffin et al found that SNM increased the amplitudes of evoked potentials in the sensory cerebral cortex during anal stimulation in a recent rat study. Following sensation of the electrophysiological experiments, the rat brains were harvested and the group also found an association between SNM and an up-regulation of the polysialylated neuronal cell adhesion molecule indicative of neuronal plasticity. ${ }^{25}$ The potential for neuromodulation during SNM is indeed intriguing. In terms of research, it might question whether PNE based and permanent SNM studies are comparable. Furthermore, it may change the future SNM treatment set-up from continuous to intermittent and may not be lifelong.

\section{Conclusion}

The positive treatment effect of SNM for IBS-D and IBS$\mathrm{M}$ is not due to an altered postprandial response. Other modes of action for the positive effect of SNM for IBS-D and IBS-M should be sought.

\section{Abbreviations}

CSA, Cross-sectional area; IBS-D, Diarrhea-predominant irritable bowel syndrome; GSRS-IBS, Gastrointestinal Symptom Rating Scale - Irritable Bowel Syndrome; IBS, Irritable Bowel Syndrome; IBS-M, Mixed irritable bowel syndrome; MPDT, Moderate pain detection threshold; PDT, Pain detection threshold; SNM, Sacral Nerve Modulation; VAS, Visual analog scale.

\section{Data Sharing Statement}

Data is available upon reasonable request attended to the corresponding author. There exists no additional unpublished data from the double-blinded, placebo-controlled crossover study.

\section{Ethics Approval and Informed Consent}

The study is approved the Danish Ethics Committee, ID110-72-170-13. All patients gave informed and written consent before enrollment in the study. 


\section{Author Contributions}

All authors have contributed with the conception and design, data acquisition, or data analysis and interpretation, drafting the article or revising it for important intellectual content, approved the final version to be published and agrees to be accountable for all aspects of the work.

\section{Funding}

Neurostimulators and leads were provided free of charge by Medtronic Inc. The company had no role in the study design, data collection, data analysis, interpretation of data, or writing of the manuscript. The Karen Elise Jensens Foundation (903959, Denmark) has financially supported Donghua Liao's work.

\section{Disclosure}

LL has previously received honoraria from Medtronic Inc. as speaker. SL has previously received an honorarium as a member of the Medtronic Inc. Medical Advisory Board. The authors report no other conflicts of interest in this work.

\section{References}

1. Thompson WG, Heaton KW, Smyth GT, Smyth C. Irritable bowel syndrome in general practice: prevalence, characteristics, and referral. Gut. 2000;46(1):78-82. doi:10.1136/gut.46.1.78

2. Palsson OS, Whitehead WE, van Tilberg MA, et al. Development and validation of the Rome IV diagnostic questionnaire for adults. Gastroenterology. 2016;150(6):1481-1491. doi:10.1053/j.gastro.2016.02. 014

3. Ragnarsson G, Bodemar G. Pain is temporally related to eating but not to defaecation in the irritable bowel syndrome (IBS). Patients' description of diarrhea, constipation and symptoms variation during a prospective 6-week study. Eur J Gastroenterol Hepatol. 1998;10 (5):415-421. doi:10.1097/00042737-199805000-00011

4. Simrén M, Månsson A, Langkilde AM, et al. Food-related gastrointestinal symptoms in the irritable bowel syndrome. Digestion. 2001;63 (2):108-115. doi:10.1159/000051878

5. Sullivan MA, Cohen S, Snape WJ. Colonical myoelectrical activity in irritable-bowel syndrome: effect of eating and anticholinergics. $N$ Engl J Med. 1978;298(16):878-883. doi:10.1056/NEJM1978 04202981604

6. Simrén M, Abrahamsson H, Björnsson ES. An exaggerated sensory component of the gastrocolic response in patients with irritable bowel syndrome. Gut. 2001;48(1):20-27. doi:10.1136/gut.48.1.20

7. Pritchard SE, Marciani L, Garsed KC, et al. Fasting and postprandial volumes of the undisturbed colon: normal values and changes in diarrhea-predominant irritable bowel syndrome measured using serial MRI. Neurogastroenterol Motil. 2014;26(1):124-130. doi:10.1111/ nmo. 12243

8. Fassov JL, Lundby L, Laurberg S, Buntzen S, Krogh K. A randomized, controlled, crossover study of sacral nerve stimulation for irritable bowel syndrome. Ann Surg. 2014;260(1):31-36. doi:10. 1097/SLA.0000000000000559
9. Fassov J, Lundby L, Laurberg S, Krogh K. Sacral nerve modulation for irritable bowel syndrome: a randomised, double-blinded, placebo-controlled crossover study. Neurogastroenterol Motil. 2019;31(6):e13570. doi:10.1111/nmo.13570

10. Fassov J, Lundby L, Laurberg S, et al. Three-year follow-up of sacral nerve stimulation for diarrhoea-predominant and mixed irritable bowel syndrome. Colorectal Dis. 2017;19(2):188-193. doi:10.1111/ codi. 13428

11. Carrington EV, Evers J, Grossi U, et al. A systematic review of sacral nerve stimulation mechanisms in the treatment of fecal incontinence and constipation. Neurogastroenterol Motil. 2014;26(9):1222-1237. doi:10.1111/nmo.12388

12. Sheldon R, Kiff ES, Clarke A, Harris ML, Hamdy S. Sacral nerve stimulation reduces corticoanal excitability in patients with faecal incontinence. Br J Surg. 2005;92(11):1423-1431. doi:10.1002/ bjs. 5111

13. Lundby L, Moller A, Buntzen S, et al. Relief of fecal incontinence by sacral nerve stimulation linked to focal brain activation. Dis Colon Rectum. 2011;54(3):318-323. doi:10.1007/DCR.0b013e31820348ac

14. Brock C, Nissen TD, Gravesen FJ, et al. Multimodal sensory testing of the rectum and the rectosigmoid: development and reproducibility of a new method. Neurogastroenterol Motil. 2008;20(8):908-918. doi:10.1111/j.1365-2982.2008.01126.x

15. Emmertsen KJ, Bregendahl S, Fassov J, Krogh K, Laurberg S. A hyperactive postprandial response in the neorectum - the clue to low anterior resection syndrome after total mesorectal excision surgery? Colorectal Dis. 2013;15(10):e599-606. doi:10.1111/codi.12360

16. Drewes AM, Frokjaer JB, Larsen E, Reddy H, Arendt-Nielsen L, Gregersen H. Pain and mechanical properties of the rectum in patients with active ulcerative colitis. Inflamm Bowel Dis. 2006;12 (4):294-303. doi:10.1097/01.MIB.0000209365.09189.04

17. Cremonini F, Houghton LA, Camilleri M, et al. Barostat testing of rectal sensation and compliance in humans: comparison of results across two centres and overall reproducibility. Neurogastroenterol Motil. 2005;17(6):810-820. doi:10.1111/j.1365-2982.2005.00709.x

18. Simrén M, Ringström G, Björnsson ES, Abrahamsson H. Treatment with hypnotherapy reduces sensory and motor component of the gastrocolonic response in irritable bowel syndrome. Psychosom Med. 2004;66(2):233-238. doi:10.1097/01.psy.0000116964.76529.6e

19. Simrén M, Simms L, D'Souza D, Abrahamsson H, Björnsson ES. Lipid-induced colonic hypersensitivity in irritable bowel syndrome: the role of 5-HT3 receptors. Aliment Pharmacol Ther. 2003;17 (2):279-287. doi:10.1046/j.1365-2036.2003.01399.x

20. Fassov J, Lundby L, Worsoe J, Buntzen S, Laurberg S, Krogh K. A randomised, controlled study of small intestinal motility in patients treated with sacral nerve stimulation for irritable bowel syndrome. BMC Gastroenterol. 2014;14:111. doi:10.1186/1471-230X-14-111

21. Fassov J, Brock C, Lundby L, et al. Sacral nerve stimulation changes rectal sensitivity and biomechanical properties in patients with irritable bowel syndrome. Neurogastroenterol Motil. 2014;26 (11):1597-1604. doi:10.1111/nmo.12426

22. Tillisch K, Mayer EA, Labus JS. Quantitative meta-analysis identifies brain regions activated during rectal distension in irritable bowel syndrome. Gastroenterology. 2011;140(1):91-100. doi:10.1053/j. gastro.2010.07.053

23. Haas S, Brock C, Krogh K, et al. Abnormal neuronal response to rectal and anal stimuli in patients with idiopathic fecal incontinence. Neurogastroenterol Motil. 2015;27(7):954-962. doi:10.1111/nmo.12567

24. Giani I, Novelli E, Martina S, et al. The effect of sacral nerve modulation on cerebral evoked potential latency in fecal incontinence and constipation. Ann Surg. 2011;254(1):90-96. doi:10.1097/ SLA.0b013e3182196ff4

25. Griffin KM, Pickering M, O'Herlihy C, O'Connell PR, Jones JFX. Sacral nerve stimulation increases activation of the primary somatosensory cortex by anal canal stimulation in an experimental model. Br J Surg. 2011;98(8):1160-1169. doi:10.1002/bjs.7536 


\section{Publish your work in this journal}

Clinical and Experimental Gastroenterology is an international, peerreviewed, open access, online journal publishing original research, reports, editorials, reviews and commentaries on all aspects of gastroenterology in the clinic and laboratory. This journal is indexed on American Chemical Society's Chemical Abstracts Service (CAS).
The manuscript management system is completely online and includes a very quick and fair peer-review system, which is all easy to use. Visit http://www.dovepress.com/testimonials.php to read real quotes from published authors. 